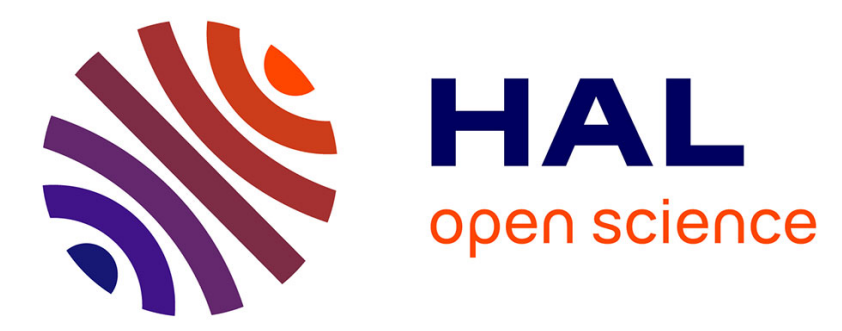

\title{
Diagnostic des machines électriques par mesure du champ magnétique rayonné. Une approche inverse
}

Viet Phuong Bui, Olivier Chadebec, Laure-Line Rouve, Jean-Louis Coulomb

\section{To cite this version:}

Viet Phuong Bui, Olivier Chadebec, Laure-Line Rouve, Jean-Louis Coulomb. Diagnostic des machines électriques par mesure du champ magnétique rayonné. Une approche inverse. European Journal of Electrical Engineering, 2008, 11 (2/3), pp.251-274. 10.3166/rige.11.287-302 . hal-03048101

\section{HAL Id: hal-03048101 https://hal.science/hal-03048101}

Submitted on 9 Dec 2020

HAL is a multi-disciplinary open access archive for the deposit and dissemination of scientific research documents, whether they are published or not. The documents may come from teaching and research institutions in France or abroad, or from public or private research centers.
L'archive ouverte pluridisciplinaire HAL, est destinée au dépôt et à la diffusion de documents scientifiques de niveau recherche, publiés ou non, émanant des établissements d'enseignement et de recherche français ou étrangers, des laboratoires publics ou privés. 


\title{
Diagnostic des machines électriques par mesure du champ magnétique rayonné
}

\author{
Une approche inverse
}

\author{
Viet Phuong Bui — Olivier Chadebec — Laure-Line Rouve \\ Jean-Louis Coulomb
}

$L E G$ - G2ELAB - UMR 5529 INPG/UJF - CNRS

ENSIEG

$B P 46$

F-38402 Grenoble cedex

olivier.chadebec@leg.ensieg.inpg.fr

\begin{abstract}
RÉSUMÉ. Cet article propose une approche originale pour la détection, la localisation et la quantification des défauts apparaissant dans les machines électriques. La technique utilisée dans ce travail repose sur l'analyse du champ magnétique rayonné (ou flux de fuite) de la machine. Cette approche est déjà connue, mais les méthodes développées jusqu'à présent permettent uniquement de statuer de la présence ou non d'un défaut. Nous proposons donc dans cet article une nouvelle approche basée sur la théorie des problèmes inverses. Celle-ci nous permet non seulement d'identifier un mode défaillant, mais également de discriminer plusieurs types de défauts, de les localiser et également de quantifier leur importance.

ABSTRACT. This paper proposes an original approach for detection, location and quantification of faults appearing in electrical machines. The used method in this work deals with the analysis of the leakage magnetic field of the machine. This approach is already known, but until now, classical methods only allow detect if a fault is present and not. Thus, we propose a new approach based on the theory of inverse problems. It not only enables us to identify a faulty mode, but also to discriminate several types of defects, to locate them and also to quantify their importance.

MOTS-CLÉS: méthode éléments finis, méthode intégrale de frontières, champ magnétique rayonné, diagnostic de machines électriques.

KEYWORDS: finite element method, boundary elements method, stray magnetic field, fault monitoring of electrical machines.
\end{abstract}




\section{Introduction}

Cet article propose une approche originale pour la détection, la localisation et la quantification des défauts apparaissant dans les machines électriques. La technique utilisée dans ce travail repose sur l'analyse du champ magnétique rayonné (ou flux de fuite) de la machine. Cette approche est déjà connue, mais les méthodes développées jusqu'à présent permettent uniquement de statuer sur la présence ou non d'un défaut. Nous proposons donc dans cet article une nouvelle approche basée sur la théorie des problèmes inverses. Celle-ci nous permet non seulement d'identifier un mode défaillant, mais également de discriminer plusieurs types de défauts, de les localiser et également de quantifier leur importance. Dans un premier temps, les potentialités de la méthode de diagnostic par mesure du flux de fuite sont brièvement exposées. Dans un deuxième temps, nous présentons plusieurs modèles permettant, à partir d'un mode de fonctionnement (défaillant ou non) de la machine, de prédire le champ magnétique à l'extérieur de celle-ci. Divers modèles plus ou moins légers sont présentés. Ensuite, nous nous intéressons à la résolution du problème inverse. Nous verrons ainsi qu'il est possible, à partir de la mesure du champ à l'extérieur de la machine d'obtenir une répartition du champ dans son entrefer. Il est alors possible d'effectuer un diagnostic dont la méthodologie est présentée en dernière partie.

\section{Champ magnétique rayonné et diagnostic de défauts des machines électriques}

\subsection{Les potentialités de l'approche}

Les dispositifs électromécaniques tiennent une place importante dans les équipements industriels (moteurs, alternateurs, actionneurs), place qui tend à s'amplifier avec l'apparition de nouvelles tendances: traction tout électrique, production décentralisée, nouveaux actionneurs, etc. De plus, ces systèmes ont souvent une place critique dans les applications. Cette criticité génère des contraintes croissantes en termes de sûreté de fonctionnement et de taux de disponibilité, nécessitant une surveillance accrue et donc le développement d'outils de diagnostic de plus en plus performants.

La première étape de la plupart des méthodes de diagnostic existantes est basée sur la connaissance du système sain (par mesures ou par modèles numériques). La deuxième étape consiste ensuite en une création «artificielle » de défauts (toujours sur maquette ou par modèles numériques). La signature du défaut, c'est-à-dire sa répercussion sur certaines grandeurs, est alors identifiée. Une observation est ensuite réalisée sur un convertisseur réel. Une analyse est portée sur les grandeurs (approche traitement du signal) consistant en la reconnaissance de signatures de défauts déjà connues.

A l'heure actuelle, la recherche de défauts est généralement effectuée sur l'analyse de grandeurs électriques que sont la tension ou l'intensité aux bornes des 
machines, de grandeurs mécaniques utiles telles que la vitesse, le couple ou par l'analyse fréquentielle de la signature des vibrations, de grandeurs thermiques par l'observation de la température de la machine (Toliyat et al., 1999).

Les approches basées sur l'analyse fréquentielle actuellement donnent de nombreux résultats significatifs mais se heurtent encore à quelques points-clés. En premier lieu, la création de défauts sur des maquettes est toujours techniquement difficile même si des méthodes de modélisation efficaces sont maintenant bien connues. De plus, l'analyse étant portée sur des grandeurs intégrées, celle-ci est souvent difficile, puisque l'information est moyennée et donc plus difficile à interpréter. Parfois, les signatures des défauts sont, en effet, très petites voire indétectables (Schmerber et al., 2005). Or, la demande croissante en terme de robustesse des outils de diagnostic nécessite de trouver de nouvelles solutions et, en particulier, de porter une analyse sur d'autres grandeurs, ceci afin de compléter les approches existantes.

Pour répondre à ces nouveaux enjeux, il est intéressant de porter l'analyse sur le champ magnétique rayonné (ou champ de fuite) par le dispositif. En effet, tout système électromécanique crée dans son environnement proche un champ magnétique. Il semble donc naturel de penser que tout défaut, intervenant à l'intérieur du système, aura nécessairement des répercussions en terme de champ rayonné. Le champ magnétique est la grandeur fondamentale de tout système électrotechnique. Il assure la conversion de l'énergie et est la cause première de tous les phénomènes physiques intervenant dans le dispositif. Tout défaut a donc, en premier lieu, des répercussions sur le champ magnétique avant de se propager sur les grandeurs que sont la tension ou l'intensité aux bornes du dispositif où son effet est moyenné et donc plus difficilement interprétable. Cette méthode possède pour nous deux grandes potentialités. D'une part, elle est non invasive. En effet, les capteurs sont situés à l'extérieur du dispositif, ce qui ne nécessite pas son démontage. D'autre part, elle peut permettre de localiser le défaut, ce que n'offrent pas les méthodes actuelles. Cette approche, encore originale, a déjà donné lieu à un certain nombre de publications (Kildishev, 2003 ; Henao et al., 2003).

\subsection{Quelques remarques qualitatives}

Dans notre approche, nous nous plaçons dans l'approximation en champ proche, à savoir que les longueurs d'ondes des champs électromagnétiques émis sont très grandes par rapport aux dimensions des dispositifs étudiés et aux distances dispositifs-capteurs. En d'autres termes, les phénomènes de propagation sont négligés dans notre étude. Cette approximation est pertinente pour les dispositifs alimentés en basses fréquences $(50 \mathrm{~Hz})$ tels que les machines électriques. Le champ magnétique alors émis par ces dispositifs peut être représenté par un modèle classique de sources multipolaires basé sur la théorie des harmoniques sphériques (Wikswo et al., 1984). 
Si nous considérons que $r$ est la distance entre le centre du dispositif et le point de mesure, l'induction mesurée en ce point est la somme de :

- un terme dipolaire (ordre 1) avec une loi de décroissance en $1 / \mathrm{r}^{3}$,

- un terme quadrupolaire (ordre 2) avec une loi de décroissance en $1 / \mathrm{r}^{4}$,

- un terme octopolaire (ordre 3 ) avec une loi de décroissance en $1 / r^{5}$,

- etc.

Il est à noter que si nous nous plaçons à une distance $\mathrm{r}$ importante, les termes d'ordres élevés ont une contribution très faible par rapport aux termes d'ordres faibles. Ceci est directement dû aux lois de décroissance des champs qui sont en $1 / \mathrm{r}^{\mathrm{n}+2}, \mathrm{n}$ étant l'ordre du terme. En particulier, suffisamment loin, seul l'ordre le moins élevé est observable. Dans le cas général, il s'agit du terme dipolaire.

Parfois ce terme peut, par nature même du dispositif, être nul. Considérons, par exemple, une machine électrique possédant 2 paires de pôles. Le terme dipolaire par raison de symétrie est nul. La machine à 4 pôles rayonne donc du champ principalement comme un quadrupôle associé à des termes d'ordres plus élevés qui deviennent négligeables à une certaine distance.

Supposons maintenant qu'un défaut apparaisse dans la machine. Celle-ci perd naturellement sa symétrie magnétique et un terme dipolaire apparaît (Chadebec et al., 2005). Cette constatation est déterminante quant à la pertinence du diagnostic par mesure du champ magnétique rayonné. En effet, si le capteur est placé suffisamment loin de la machine, seul le terme dipolaire est mesuré, or dans le cas de la machine saine celui-ci est en théorie nul. Si un défaut apparaît, le terme dipolaire devient plus important et le champ mesuré par le capteur également. Ceci permet de démontrer que la méthode est attractive puisque, par nature, les défauts des machines électriques ont tendance à faire augmenter d'une façon très importante leurs champs de fuite.

\section{Calcul du champ magnétique rayonné par une machine électrique}

\subsection{Introduction au calcul du champ rayonné par les machines électriques}

Le calcul du champ magnétique rayonné à l'extérieur d'une machine est un problème relativement difficile. En effet, celui-ci est fortement $3 \mathrm{D}$ et nécessite donc la mise en œuvre d'une modélisation très lourde. De plus, la forte disproportion entre le champ à l'intérieur de la machine et le champ à l'extérieur (généralement d'un rapport $10^{6}$, à savoir 1-2T dans l'entrefer et de l'ordre du $\mu \mathrm{T}$ à l'extérieur) renforce la difficulté de la modélisation, le modélisateur étant confronté au problème de soustraction de grands nombres. Enfin, le problème est ouvert (région air extérieur infinie), ce qui rajoute une difficulté de modélisation. Il est donc à noter que ce type de modélisation conduit à des difficultés plus importantes que celles que l'on rencontre lorsqu'on modélise, d'une façon très classique, une machine électrique en $2 \mathrm{D}$. 


\subsection{La méthode éléments finis}

La méthode privilégiée pour calculer le champ rayonné par une machine électrique est traditionnellement la méthode éléments finis. Il convient de tronquer artificiellement la région air entourant la machine. Afin de garder un domaine de taille raisonnable et donc un nombre d'éléments admissibles, une bonne solution est d'utiliser le concept de "boîte infinie » qui modélise la région dite infinie par transformation (Brunotte et al., 1992).

Pourtant, cette approche n'est qu'imparfaite. En effet, le calcul du champ dans l'air est sujet à des imprécisions dues au bruit de maillage. Ce problème peut être contourné en le raffinant mais nous sommes rapidement confrontés à des problèmes dont la taille explose. Une solution peut être de modéliser la région air extérieur par méthode intégrales de frontières. Il convient alors de réaliser un couplage avec la méthode éléments finis à l'interface air-fer. Pourtant, cette approche est rapidement limitée avec l'apparition de bloc matrice plein dans le système final à résoudre, ce qui pose de nouvelles difficultés.

Une approche développée dans notre laboratoire semble, à notre sens, un bon compromis. Il s'agit d'effectuer une modélisation classique 3D de la machine par la méthode des éléments finis en utilisant la technique de la boîte infinie. Une fois le problème résolu, l'aimantation $\mathbf{M}$ des matériaux ferromagnétiques peut être calculée aisément. Il est alors possible de calculer le champ rayonné par la machine en appliquant un postprocessing basé sur une équation intégrale de volume. Cette méthode est généralement connue sous le nom de méthode des moments. Le champ magnétique à l'extérieur du dispositif a alors pour expression :

$$
\mathbf{B}=\frac{\mu_{0}}{4 \pi} \iiint_{V_{\operatorname{mag}}}\left(3 \frac{\mathbf{M} \cdot \mathbf{r}}{r^{5}} r-\frac{\mathbf{M}}{r^{3}}\right) d V+\mathbf{B}_{\mathbf{0}}
$$

où $\mathrm{V}_{\text {mag }}$ est le volume de matériau ferromagnétique, $\mathbf{M}$ est l'aimantation de ce volume calculé par élément finis, $\mathbf{r}$ est le vecteur reliant le point d'intégration au point où est calculé le champ, $\mathbf{B}_{\mathbf{0}}$ est le champ créé par les bobinages dans l'air.

Cette méthode a été validée expérimentalement dans notre laboratoire (Froidurot et al., 2002). Plusieurs types de machine synchrone (flux transverse, flux axial) ont été instrumentés, dans le simulateur de champ du LMMCF (Laboratoire de métrologie magnétique en champs faibles), avec l'aide de magnétomètres du type fluxgate. Le simulateur de champ permettait de travailler dans un environnement magnétique parfaitement contrôlé, de plus, la technologie fluxgate permet une très bonne précision de mesure. Le dispositif a permis ainsi de valider l'approche éléments finis couplée avec une exploitation intégrale de volume.

Pourtant, cette approche demeure lourde à mettre en œuvre. En particulier, comme nous l'avons évoqué précédemment, pour le cas de fonctionnement en mode 
défaillance, un moteur électrique perd ses symétries naturelles. Il est alors nécessaire de mailler toute sa géométrie, ce qui pose encore des problèmes en terme de taille mémoire.

Nous avons donc décidé de développer un modèle simplifié basé sur une homogénéisation. Cette approche est très classique mais est, dans le cadre de ce travail, originalement appliquée à la problématique du calcul du champ magnétique rayonné par les machines. Elle est présentée dans la section suivante.

\subsection{Approche homogénéisée}

Le but de cette section est de présenter une approche permettant de réduire significativement la taille des maillages associés à une modélisation 3D éléments finis d'une machine électrique, tout en gardant un champ magnétique externe calculé précisément. Dans ce type de modélisation, c'est généralement le maillage des encoches qui est très gourmand en nombre d'éléments. Nous nous proposons donc de remplacer les encoches par un matériau magnétique anisotrope équivalent. Le maillage comportera donc moins d'éléments et la modélisation de la machine complète avec défaut sera alors envisageable.

La principale approximation de notre approche réside dans l'idéalisation de la distribution d'induction à l'intérieur de la machine. En effet, les lignes de champs sont radiales dans l'entrefer et dans les zones délimitées par les encoches du rotor et $\mathrm{du}$ stator. Pour représenter le comportement de l'induction dans les dents, considérons la figure 1 , où $B_{d}$ et $B_{\text {enc }}$ sont respectivement les valeurs moyennes de l'induction dans la dent et dans l'encoche.

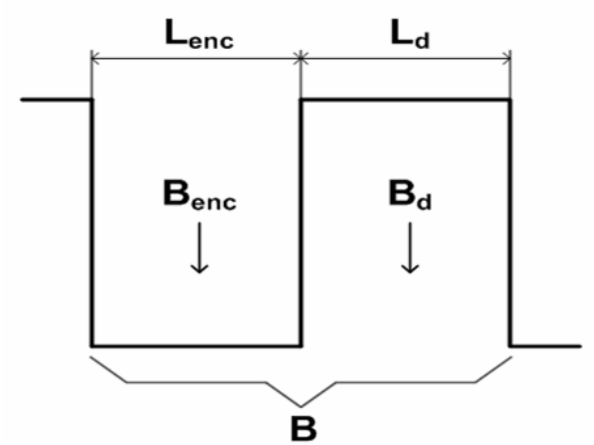

Figure 1. Représentation d'une encoche et d'une dent et inductions associées

L'induction magnétique moyennée B sur la dent et l'encoche est alors donnée par l'expression suivante : 


$$
\mathrm{B}(\mathrm{H})=\frac{\mathrm{B}_{\mathrm{d}}(\mathrm{H}) \times \mathrm{L}_{\mathrm{t}}+\mathrm{B}_{\mathrm{enc}}(\mathrm{H}) \times \mathrm{L}_{\mathrm{s}}}{\mathrm{L}_{\mathrm{d}}+\mathrm{L}_{\mathrm{enc}}}
$$

où $\mathrm{L}_{\mathrm{d}}$ et $\mathrm{L}_{\mathrm{enc}}$ sont respectivement les largeurs des dents et des encoches. Appelons $\mathbf{H}$ le champ magnétique dans le fer et dans le vide associé à l'induction moyennée. Si la caractéristique $\mathrm{B}_{\mathrm{d}}(\mathrm{H})$ du matériau est connue, il est possible de remplacer la région constituée des dents et des encoches par une région homogénéisée donc les caractéristiques magnétiques $\mathrm{B}(\mathrm{H})$ sont données par la formule suivante :

$$
\mathrm{B}(\mathrm{H})=\frac{\mathrm{B}_{\mathrm{d}}(\mathrm{H}) \times \mathrm{L}_{\mathrm{d}}+\mu_{0} \times \mathrm{H} \times \mathrm{L}_{\mathrm{enc}}}{\mathrm{L}_{\mathrm{d}}+\mathrm{L}_{\mathrm{enc}}}
$$

Dans le cas d'une loi de matériau linéaire, l'équation [3] conduit à une perméabilité relative équivalente. Cette perméabilité est anisotrope et les sens de facile aimantation sont la direction radiale et la direction axiale du moteur.

Les perméabilités relatives radiale $\mu_{\mathrm{r} 1}$ et axiale $\mu_{\mathrm{r} 3}$ ont pour expression :

$$
\mu_{\mathrm{r} 1}=\mu_{\mathrm{r} 3}=\frac{\mu_{\mathrm{r}} \times \mathrm{L}_{\mathrm{d}}+\mathrm{L}_{\mathrm{enc}}}{\mathrm{L}_{\mathrm{d}}+\mathrm{L}_{\mathrm{enc}}}
$$

La perméabilité relative orthoradiale $\mu_{\mathrm{r} 2}$ est :

$$
\mu_{\mathrm{r} 2}=\frac{\mathrm{L}_{\mathrm{d}}+\mathrm{L}_{\mathrm{enc}}}{\mathrm{L}_{\mathrm{enc}}}
$$

Celle-ci est très proche de 1 et peut ne pas être prise en compte.

Grâce à cette approche, un maillage léger peut être utilisé pour mailler le moteur puisque la géométrie des encoches n'est plus prise en compte. Le nombre d'inconnues est beaucoup moins important et un maillage de toute la géométrie est envisageable. Il est à noter que le modèle ne prenant pas en compte les encoches, les harmoniques du champ rayonné dues à celles-ci ne sont naturellement plus calculées. Nous verrons que ce point n'est pas réellement bloquant pour notre approche. De plus, il est nécessaire de signaler qu'une fois le calcul éléments finis effectué, il est toujours nécessaire d'effectuer une exploitation par méthode intégrale de volume. Les résultats de cette approche seront présentés dans la section 3.5.

\subsection{Un modèle simplifié : le modèle entrefer surfacique}

Nous avons vu dans les sections précédentes qu'une modélisation éléments finis 3D de machines électriques avec défauts dans l'optique de calculer le champ magnétique rayonné était envisageable. Pourtant, même avec la simplification apportée par l'homogénéisation, l'approche éléments finis demeure lourde à 
manipuler. Devant cette relative lourdeur, nous avons décidé de concentrer nos efforts sur le développement d'une approche alternative, plus légère, dont le but est d'une part de calculer rapidement le champ rayonné par un moteur électrique et d'autre part de pouvoir être inversée.

Notons que pour cette méthode, nous considérons que le matériau est linéaire, ce qui n'est pas nécessairement le cas dans le modèle éléments finis homogénéisé. Cette approximation peut être considérée comme simpliste, il faut pourtant faire remarquer que ces travaux sont en phase d'initiation et que le modèle développé est original. A notre sens, son extension pour le cas non linéaire est parfaitement envisageable mais simplement plus complexe à mettre en œuvre.

\subsubsection{Quelques remarques préliminaires}

\subsubsection{Un modèle dipôle normal équivalent du bobinage}

Considérons une boucle de courant délimitée par une courbe $\mathrm{C}$ dans laquelle circule un courant I. Le champ créé par cette boucle est exactement le même que celui créé par toute surface $\mathrm{S}$ s'appuyant sur $\mathrm{C}$ et portant une distribution normale de dipôles dont la densité surfacique est I.n, où $\mathbf{n}$ est la normale à $\mathrm{S}$ (Durant, 1969). Il est aisé de comprendre en examinant la figure 2 qu'une telle surface est équivalente à la courbe $\mathrm{C}$ portant un courant. En effet, nous considérons un réseau de petites boucles infinitésimales de courant (donc des dipôles) dont la surface tend vers 0 et dont les contributions se compensent naturellement dans la surface sauf sur la courbe C la délimitant (figure 2).

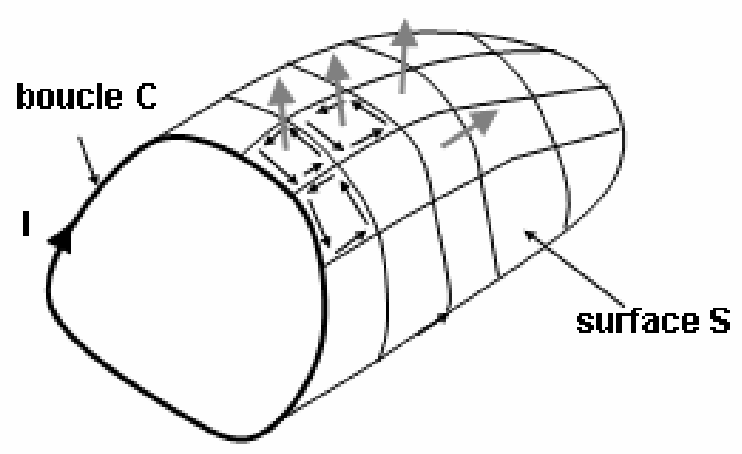

Figure 2. Equivalence entre un courant circulant dans une boucle et une densité surfacique de dipôles normaux

\subsubsection{Un modèle dipolaire équivalent pour la matière aimantée}

Considérons maintenant un volume $\mathrm{V}$ uniformément aimanté par une aimantation M délimité par une surface $\mathrm{S}$. Ce volume aimanté crée exactement le même champ 
qu'une densité de charge surfacique de valeur M.n, où $\mathbf{n}$ est la normale sortante à $\mathrm{V}$ et portée par $\mathrm{S}$. Ce résultat est basé sur les équivalences ampériennes/coulombiennes (Durand, 1968). Une représentation en est donnée figure 3.
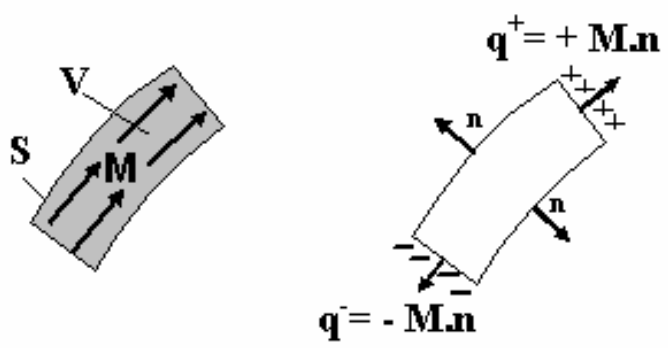

Figure 3. Equivalence entre un volume uniformément aimanté et une densité surfacique de charges

\subsubsection{Le modèle entrefer surfacique}

Notre modèle simplifié repose sur l'application des deux équivalences précédentes à la géométrie d'un moteur. Considérons, pour expliciter notre approche, une machine électrique composée d'une paire de pôles, d'un rotor et d'un stator. Dans cette approche, comme dans l'approche homogénéisée, la géométrie des encoches n'est pas prise en compte.

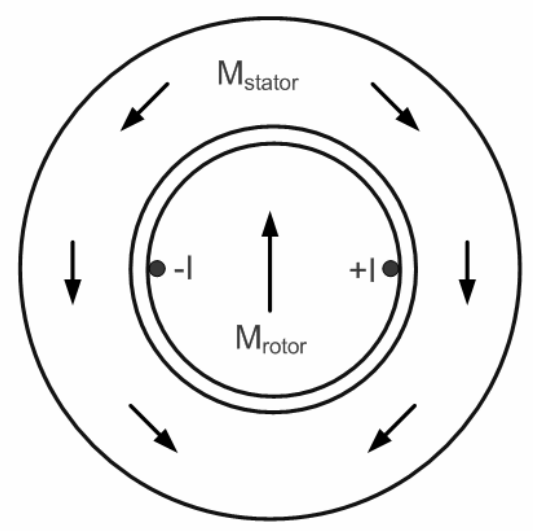

Figure 4. Représentation simplifiée d'une machine électrique à une paire de pôles. $\boldsymbol{M}_{\text {stator }}$ et $\boldsymbol{M}_{\text {rotor }}$ sont les aimantations des pièces ferromagnétiques

Le champ rayonné par la machine est la somme du champ créé par le bobinage et du champ créé par la matière aimantée. 
D'après la première équivalence (section 3.4.1.1.), le champ créé par le bobinage est le même que celui créé par une distribution surfacique de dipôles que nous nommerons $\mathbf{T}_{\mathbf{0}}$ et que l'on peut localiser sur une surface moyenne de l'entrefer (voir figure 5). La valeur de la répartition est égale au nombre d'ampères-tours créés par le bobinage au point considéré. Notons que si l'on connaît parfaitement le bobinage, la densité surfacique $\mathbf{T}_{\mathbf{0}}$ est parfaitement connue et calculable.

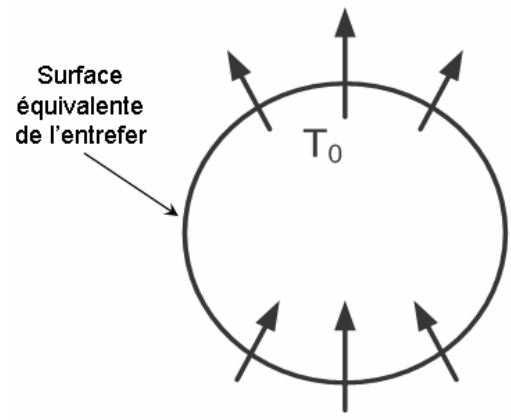

Figure 5. Représentation d'une distribution surfacique de dipôles normaux dans l'entrefer

D'après la deuxième équivalence (section 3.4.1.2.), le champ créé par la matière est le même que celui créé par trois densités surfaciques de charges, réparties de la façon suivante :

- une densité surfacique de charges localisée sur la frontière entre le rotor et l'entrefer. Cette densité de charge a pour valeur $\mathbf{M}_{\text {rotor }} \mathbf{n}$, où $n$ est la normale sortante du rotor vers l'entrefer,

- une densité surfacique de charges localisée sur la frontière entre le stator et l'entrefer. Cette densité de charge a pour valeur $\mathbf{M}_{\text {stator }} \mathbf{n}$, où $n$ est la normale sortante du stator vers l'entrefer. Elle a une direction opposée à la normale précédente,

- une densité surfacique de charges localisée sur la frontière entre le stator et la région extérieure. Cette densité de charges représente les fuites passant du stator vers l'air.

A ce stade, une première remarque s'impose. Considérons les deux premières distributions. Le champ dans l'entrefer de la machine est radial. Les deux surfaces entrefer/rotor et stator/entrefer portent donc des distributions surfaciques de charges dont les densités sont quasiment les mêmes, à un signe près dû à la différence d'orientation des normales. Comme les charges ont la même valeur à un signe près, on peut les représenter par une distribution dipolaire $\mathbf{T}_{\text {mat }}$ localisée sur la surface moyenne de l'entrefer (figure 6) et de valeur :

$$
\mathrm{T}_{\text {mat }}=\mathrm{e} \times \frac{1}{\mu_{0}} \times \mathrm{B}_{\text {entrefer }}
$$


où e est l'épaisseur de l'entrefer, $\mu_{0}$ est la perméabilité du vide et $B_{\text {entrefer }}$ est le champ circulant dans l'entrefer au point considéré.

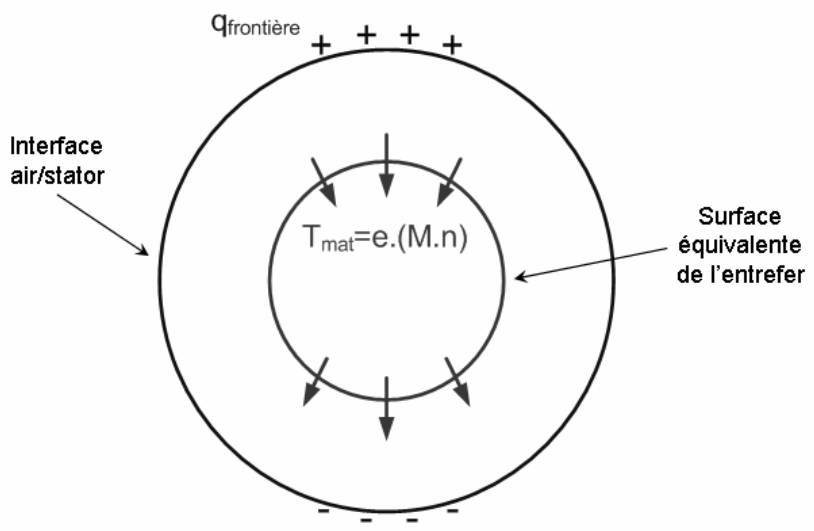

Figure 6. Représentation des distributions surfaciques de dipôles normaux dans l'entrefer et charges à l'extérieur

Les distributions $\mathbf{T}_{\mathbf{0}}$ et $\mathbf{T}_{\text {mat }}$ sont toutes les deux localisées sur la surface moyenne de l'entrefer, appelons $\mathbf{T}$ la somme de ces deux distributions. Le champ créé par la machine est donc la somme du champ créé par la distribution $\mathbf{T}$ dans l'entrefer et la distribution q localisée sur l'interface stator-air (voir figure 7).

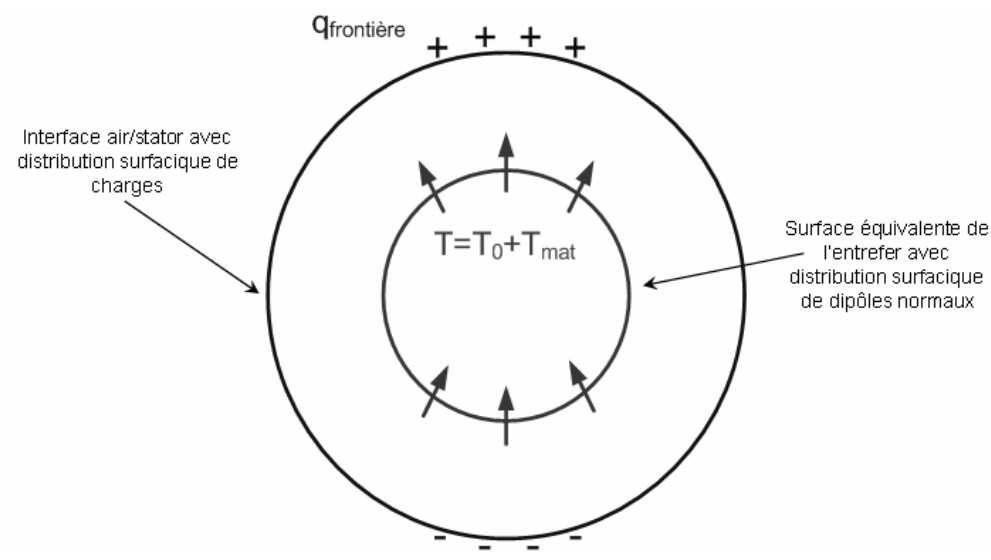

Figure 7. Représentation simplifiée d'une machine électrique à une paire de pôles. Le champ externe est créé par la distribution q et $\boldsymbol{T}$ 


\subsubsection{La détermination du champ dans l'entrefer}

Il convient maintenant d'expliciter comment peut être calculé $\mathbf{T}_{\text {mat }}$. Remarquons que cette grandeur dépend de l'induction dans l'entrefer. Si nous connaissons cette induction, nous connaissons la valeur de la densité dipolaire.

Pour une machine ayant un comportement sain, le champ circulant dans l'entrefer est une grandeur de conception. Il est toujours connu puisque c'est généralement la grandeur de départ qui a conduit aux paramètres géométriques définissant la structure de la machine. Cette grandeur est donc dans la plupart des cas maîtrisée et a une expression analytique connue dépendant du temps et de l'angle spatial.

Pour un fonctionnement avec défaut, plusieurs stratégies sont possibles. Il est possible, pour connaître le champ, de réaliser un modèle réseau de reluctance de la machine électrique. Ce modèle peut permettre d'introduire différents types de défauts et d'obtenir la répercussion de ceux-ci sur le champ dans l'entrefer (Jérance, 2002). D'une manière plus générale, un bon nombre d'ouvrages traite de l'influence des défauts sur le champ dans l'entrefer (Timar, 1989).

Par exemple, pour une machine synchrone à vide, nous avons comme terme principal :

$$
\mathrm{B}(\theta, \mathrm{t})=\mathrm{B}_{\max } \times \cos \left(\mathrm{p} \theta-\omega_{\mathrm{s}} \mathrm{t}\right)
$$

où $\mathrm{B}_{\max }$ représente l'amplitude du champ dans l'entrefer, $\mathrm{p}$ le nombre de paires de pôles, $\omega_{\mathrm{s}}$ la pulsation de synchronisme et $\mathrm{t}$ le temps. Si un court-circuit apparaît, l'induction est principalement modifiée selon :

$$
\mathrm{B}(\theta, \mathrm{t})=\mathrm{B}_{\max } \times \cos \left(\mathrm{p} \theta-\omega_{\mathrm{s}} \mathrm{t}\right)+\lambda_{\mathrm{cc}} \times \cos \left(\theta-\omega_{\mathrm{s}} \mathrm{t} / \mathrm{p}\right)
$$

où $\lambda_{\mathrm{cc}}$ représente l'amplitude du défaut de court-circuit. Dans le cas d'une excentricité dynamique, nous aurons également :

$$
\mathrm{B}(\theta, \mathrm{t})=\mathrm{B}_{\max } \times \cos \left(\mathrm{p} \theta-\omega_{\mathrm{s}} \mathrm{t}\right) \times\left(1+\lambda_{\text {de }} \times \sin \left(\theta-\omega_{\mathrm{s}} \mathrm{t} / \mathrm{p}\right)\right)
$$

où $\lambda_{\text {de }}$ représente l'amplitude de l'excentricité.

Nous avons donc vu qu'il était possible, en connaissant bien le mode de fonctionnement de la machine, de déterminer analytiquement la valeur de $\mathbf{T}_{\text {mat }}$. La valeur $\mathbf{T}_{\mathbf{0}}$ est, comme nous l'avons vu, elle aussi calculable. La valeur totale $\mathbf{T}$ a donc une expression analytique connue dans un bon nombre de cas. Il convient donc maintenant de déterminer la dernière quantité manquante, à savoir la distribution $\mathrm{q}$ de charges localisées à l'interface stator-air. 


\subsubsection{Détermination de $q$}

Nous assimilons maintenant notre machine à un cylindre plein de matériau ferromagnétique. A l'intérieur de ce cylindre, il existe une distribution de sources dipolaires localisée sur une surface représentant l'entrefer. Cette distribution, connue, crée un champ $\mathbf{B}_{\mathbf{0}}$ partout dans l'espace. Le matériau ferromagnétique canalise principalement l'induction mais une petite partie de celle-ci s'échappe dans l'air. Ces fuites peuvent être représentées par la densité de charges q localisée à la frontière entre le cylindre plein et l'air. Il est possible, dans le cas d'un matériau linéaire de calculer cette répartition q par une méthode intégrale bien connue. La distribution q vérifie l'équation intégrale écrite sur $\mathrm{S}$, la surface frontière entre le cylindre et l'air, suivant :

$$
\frac{\mu_{\mathrm{r}}+1}{\mu_{\mathrm{r}}-1} \mathrm{q}-\frac{1}{2 \pi} \iint_{\mathrm{S}} \mathrm{q} \frac{\mathbf{r} \cdot \mathbf{n}}{\mathrm{r}^{3}} \mathrm{~d} \mathrm{~S}=2\left(\mathbf{n} \cdot \mathbf{B}_{\mathbf{0}}\right)
$$

où $\mu_{\mathrm{r}}$ est la perméabilité relative du matériau ferromagnétique, $\mathbf{r}$ est le vecteur reliant le point où est écrit l'équation au point d'intégration, $\mathbf{n}$ est la normale sortante cylindre, $\mathbf{B}_{0}$ est l'induction crée par la distribution dipolaire sur $\mathrm{S}$.

Il suffit alors de discrétiser $\mathrm{S}$ et de résoudre [10] par une simple collocation, pour écrire un système linéaire qu'il suffit de résoudre pour obtenir q. Le champ à l'extérieur de la machine a alors pour expression :

$$
\mathrm{B}=\frac{\mu_{0}}{4 \pi} \iint_{S_{\text {entrefer }}}\left(3 \frac{T \cdot r}{r^{5}} r-\frac{T}{r^{3}}\right) d S+\frac{\mu_{0}}{4 \pi} \iint_{S_{\text {stator /air }}} \mathrm{q} \frac{\mathrm{r}}{\mathrm{r}^{3}} \mathrm{dS}
$$

où $\mathbf{T}$ est connu, puisque nous connaissons son expression spatiale et q est déterminé par la résolution de [10].

REMARQUE. - Ce modèle présente l'inconvénient de n'être applicable qu'aux matériaux linéaires. En effet, l'approche que nous proposons est nouvelle et originale puisque, à notre connaissance, aucun modèle similaire n'existe dans la littérature. Il faut savoir que cette approche peut être généralisée en non linéaire, l'équation [10] devenant une intégrale de volume. Celle-ci peut alors être résolue par une approche nommée méthode des moments (Chadebec et al., 2006) et un procédé itératif. Nous n'avons pourtant pas testé cette approche par manque de temps. Elle le sera prochainement. Remarquons également que la méthodologie que nous proposons est très générale et peut être adaptée facilement pour calculer le champ externe généré par d'autres types de structures (transformateurs avec entrefer, contacteurs...). 


\subsubsection{Prise en compte de la carcasse et des masses magnétiques externes}

L'approche présentée ne prend en compte que l'ensemble bobinage, rotor et stator. Il est à noter que, généralement, les machines électriques sont associées à d'autres masses ferromagnétiques, en particulier des objets voisins (armoire...) indépendants du dispositif sous diagnostic. L'exemple le plus courant est bien entendu la carcasse de la machine, qui joue un rôle électromagnétique important en blindant le champ créé par la machine seule. Notre approche peut facilement être couplée avec une méthode du type moment magnétique (Chadebec et al., 2006) particulièrement bien adaptée pour calculer l'effet de blindage statique. Il est en effet possible de relier l'aimantation de la carcasse aux grandeurs $\mathbf{T}$ et q, par l'équation intégrale de volume suivante :

$$
M-\left(\mu_{2 r}-1\right) \iiint_{V_{\text {carcasse }}} \frac{M \cdot r}{r^{3}} d V=\left(\mu_{2 r}-1\right) H_{0}(T, q)
$$

où $\mathbf{M}$ est l'aimantation de la carcasse, $\mathbf{r}$ est le vecteur reliant le point où est écrit l'équation au point d'intégration, $\mu_{2 r}$ est la perméabilité de la carcasse et $\mathbf{H}_{\mathbf{0}}$ est le champ créé par les distributions $\mathbf{T}$ et q précédentes. La prise en compte des interactions est possible en résolvant simultanément [10] et [12] dans un unique système pour obtenir à partir de $\mathbf{T}$, les distributions q et $\mathbf{M}$. Il est alors possible de calculer le champ à l'extérieur de la machine en prenant en compte l'effet de blindage de la carcasse ou même l'effet d'autres masses ferromagnétiques.

Notons que la prise en compte des effets de blindages dynamiques (courants induits dans la carcasse) doit donner lieu à des développements supplémentaires que nous n'abordons pas ici.

\subsection{Comparaisons des modèles}

Nous avons testé nos trois approches (éléments finis + postprocessing avec intégrale de volume, éléments finis homogénéisés + postprocessing avec intégrale de volume et modèle entrefer surfacique) sur une machine synchrone à 2 paires de pôles et de $30 \mathrm{~kW}$, disponible dans notre laboratoire. La machine est sans défaut et présente donc symétries et périodicités. Par simplicité de représentation, la carcasse n'est pas modélisée ici. 
Le modèle éléments finis incluant la description des encoches est le suivant :

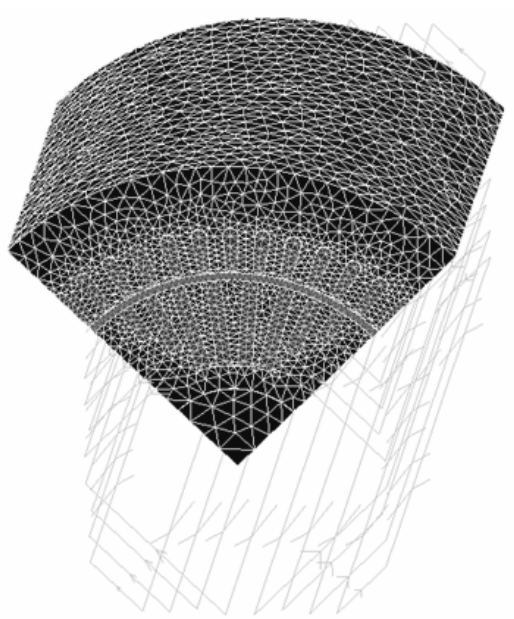

Figure 8. Modèle éléments finis $3 D$ maillé en prenant en compte les encoches. 1/8 de la machine est représenté avec 594482 éléments

De même, le modèle équivalent homogénéisé est mis en œuvre. Le nombre d'éléments est considérablement réduit.

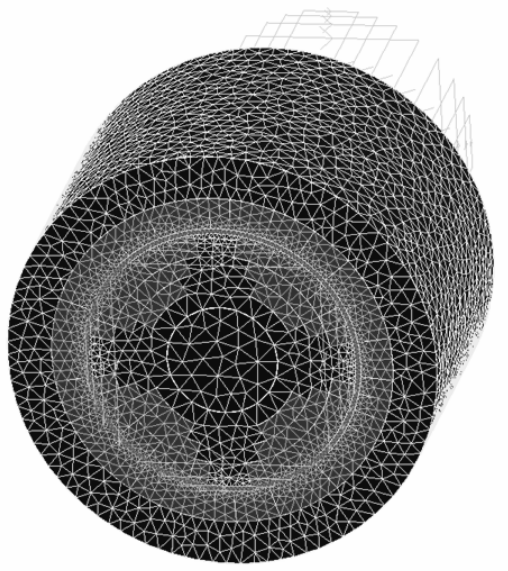

Figure 9. Modèle éléments finis $3 D$ homogénéisé. 1/2 de la machine est représenté avec 546720 éléments 
Enfin le modèle entrefer surfacique est lui aussi mis en œuvre. 600 éléments surfaciques sont alors nécessaires.

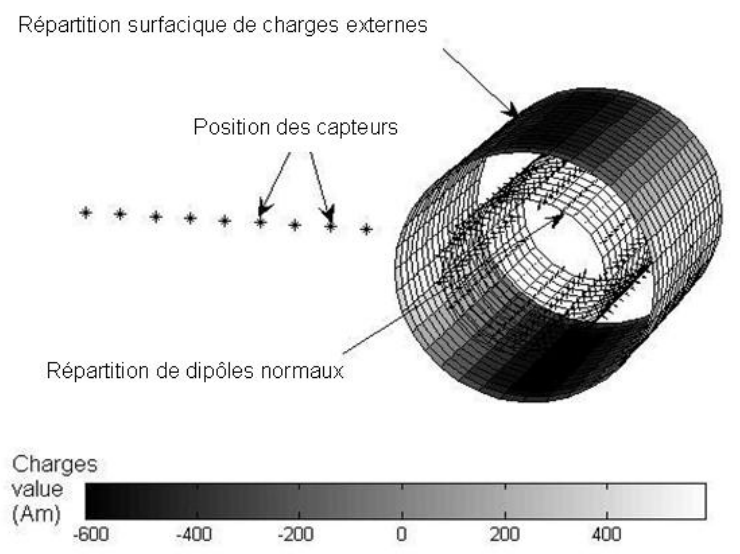

Figure 10. Modèle entrefer surfacique. Le cylindre interne porte les dipôles normaux $\boldsymbol{T}$, le cylindre externe les charges $q$

Les champs calculés sur des capteurs localisés à l'extérieur de la machine (figure 10) sont comparés pour les trois approches.

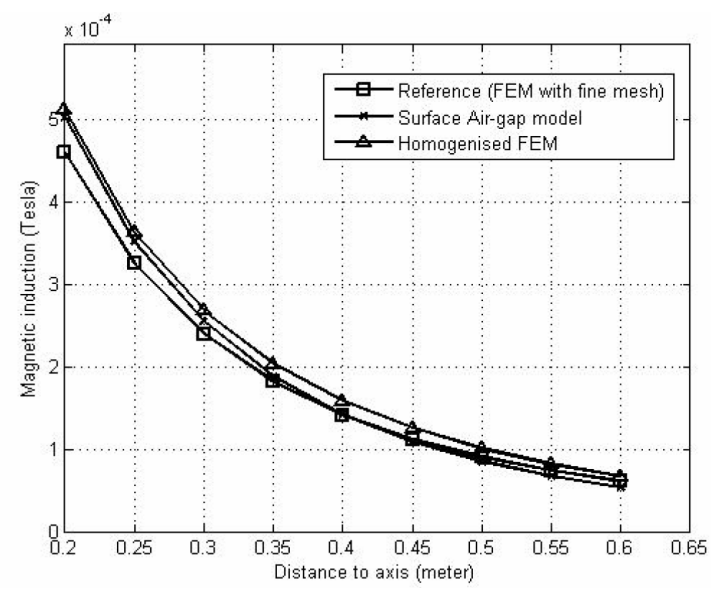

Figure 11. Comparaison des champs calculés par les trois modèles sur un ensemble de capteurs placés à l'extérieur de la machine. Seule la composante radiale du champ est représentée 
Les courbes de la figure 11 présentent une bonne adéquation. D'un point de vue général, l'erreur retournée par le modèle homogénéisé n'excède pas les $5 \%$, tandis que l'erreur du modèle entrefer surfacique n'excède pas $20 \%$. Ceci peut être considéré comme admissible quand on se rappelle le gain considérable sur le nombre d'éléments utilisés dans notre modèle simplifié.

Il nous est donc maintenant possible de prévoir le champ rayonné par une machine fonctionnant en mode défaillant. En effet, l'approche homogénéisée permet à présent de modéliser une machine complète, ce qui était impossible avec un modèle prenant en compte les encoches. De plus, le modèle surfacique permet un calcul similaire avec un nombre d'inconnues drastiquement réduit. Nous allons voir que non seulement cette approche est légère mais de plus elle possède l'énorme avantage d'être inversible.

\section{Résolution du problème inverse}

Une approche classique en modélisation consiste, à partir de la connaissance des sources, de déterminer les effets. Dans la partie précédente, les sources de champ étaient essentiellement dues au bobinage et au flux circulant dans l'entrefer. Celles-ci ont été regroupées sous la grandeur $\mathbf{T}$. Il est ensuite possible, à partir de la connaissance de ces sources, de calculer les effets, à savoir, pour le cas qui nous intéresse, le champ rayonné. Dans cette partie, nous allons résoudre le problème inverse. Nous allons, à partir de la mesure du champ réalisée sur un réseau de capteurs, tenter de retrouver une image de l'induction dans l'entrefer, à savoir la répartition $\mathbf{T}$. Une fois cette répartition obtenue, nous pourrons alors localiser le défaut et mieux encore quantifier son importance.

\subsection{Ecriture de la relation champ entrefer/champ rayonné}

Pour résoudre notre problème inverse, il convient, tout d'abord, d'établir la relation liant le vecteur $\mathbf{T}$ au champ externe mesuré $\mathbf{B}_{\text {mes. }}$. L'équation [10] peut se traduire matriciellement de la façon suivante :

$$
[\mathrm{U}] \mathbf{q}=[\mathrm{V}] \mathbf{T}
$$

De plus, l'équation [11] peut elle aussi s'écrire matriciellement :

$$
\left[\mathrm{S}_{\mathrm{T}}\right] \mathbf{T}+\left[\mathrm{S}_{\mathrm{q}}\right] \mathbf{q}=\mathbf{B}_{\text {mes }}
$$

En combinant [13] et [14], nous obtenons la matrice $\mathbf{A}$ qui relie les sources $\mathbf{T}$ au champ mesuré $\mathbf{B}_{\text {mes. }}$. 


$$
[\mathrm{A}] \mathbf{T}=\left[\mathrm{S}_{\mathrm{T}}+\mathrm{S}_{\mathrm{q}} \mathrm{U}^{-1} \mathrm{~V}\right] \mathbf{T}=\mathbf{B}_{\mathbf{m e s}}
$$

Cette matrice présente $3 \mathrm{k}$ lignes, si $\mathrm{k}$ est le nombre de capteurs et si les mesures sont vectorielles, pour $\mathrm{p}$ colonnes, où $\mathrm{p}$ est le nombre d'éléments choisi pour représenter l'entrefer. Remarquons que si la machine possède une carcasse, une équation similaire à [15] peut également être écrite. Elle est simplement un peu plus complexe.

\subsection{Inversion de la relation}

\subsubsection{Généralités}

Plaçons donc k capteurs autour de la machine électrique.

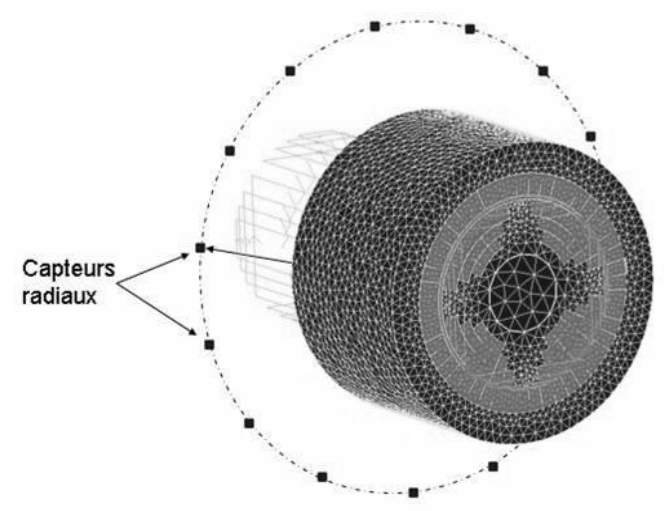

Figure 12. Positionnement des capteurs autour de la machine électrique pour résolution du problème inverse

Typiquement, ce problème inverse sera mal posé. Cette caractéristique est intrinsèque à la nature même des problèmes inverses et se traduit par une solution non unique. Ce caractère mal posé se traduira par un conditionnement de A très mauvais et par l'obtention de solutions généralement numériquement divergentes. Une solution très classique est d'utiliser un procédé de régularisation de Tikhonov pour éviter ces divergences. Pourtant, nous avons préféré utiliser une solution plus subtile qui consiste à utiliser une base optimale (c'est-à-dire orthogonale) pour la description de T. Cette stratégie est, elle aussi, largement utilisée comme méthode de résolution des problèmes inverses et conduit généralement à des matrices $\mathrm{A}$ bien conditionnées. 


\subsubsection{Une base optimale pour $T$}

Le champ dans l'entrefer, et donc la distribution $\mathrm{T}$, peut être décrit par une décomposition harmonique spatio-temporelle suivant la formule :

$$
\mathrm{T}(\theta, \mathrm{t})=\langle\mathrm{T}\rangle+\sum_{\mathrm{i}=1}^{\mathrm{p}} \mathrm{T}_{\mathrm{i}} \cos \left(\mathrm{i} \theta-\omega_{\mathrm{i}} \mathrm{t}\right)
$$

où $<\mathrm{T}>$ représente la valeur moyenne de $\mathrm{T}$. En théorie, $\mathrm{p}$ doit être infini mais on peut tronquer la série tout en gardant une précision admissible (dans l'exemple suivant $\mathrm{p}$ sera pris égal à 4). Cette base possède évidemment un lien avec la théorie des séries de Fourier. Elle possède donc l'intérêt d'être orthogonale sur la surface de l'entrefer. Il n'est donc pas abusif de penser qu'elle présentera également des propriétés d'orthogonalité dans l'espace des champs rayonnés.

Considérons les bases de $\mathrm{T}$ correspondant à $(\mathrm{i}=1,2,3,4)$. Nous appliquons $\mathrm{p}$ fois le système [15] et nous obtenons ainsi $p$ vecteurs intervenant dans la décomposition du champ rayonné.

Considérons la base $<\mathrm{T}>$, celle-ci représente une distribution de dipôles uniforme dans l'entrefer. Si nous nous reportons à la figure 2, il est aisé de voir que le champ créé par cette base correspond à celui créé par deux boucles localisées aux têtes de bobines et parcourues par des courants de sens opposé. Cette base représente donc une rupture d'antipériodicité au niveau des têtes de bobines.

Supposons maintenant que nous effectuons nos mesures sur notre réseau de capteurs. Nous obtenons 3.k informations qui correspondent à la dimension du vecteur $\mathrm{B}_{\text {mes }}$ (les capteurs sont tri-axes). Nous avons alors $(\mathrm{p}+1)$ inconnues à déterminer (les valeurs de $T_{i}$ et $\langle T>$ ). Nous pouvons donc écrire notre système linéaire reliant ces coefficients à $\mathrm{B}_{\text {mes. }}$. Le système est bien conditionné puisque la base est optimale, il suffit alors de le résoudre par une pseudo-inversion basée sur une décomposition en valeurs singulières.

\section{Diagnostic de défauts}

Nous nous proposons de tester notre approche sur une machine synchrone ( $30 \mathrm{~kW}, 2$ paires de pôles). Dans cet article, nous étudions une configuration simple, à savoir en alternateur fonctionnant à vide. Dans un premier temps, nous montrerons que notre approche permet de discriminer les défauts, puis nous montrerons qu'elle peut également permettre de les localiser et de les quantifier.

La méthodologie employée est la suivante. Dans un premier temps, nous modélisons notre machine avec défaut par la méthode des éléments finis (voir sections 3.2. et 3.3.). Ce calcul permet d'évaluer le champ magnétique mesuré par les capteurs localisés à l'extérieur de la machine. A partir de ces mesures « virtuelles » obtenues, nous inversons le système [15] pour obtenir la répartition de 
dipôles représentative du champ dans l'entrefer. Il est ensuite possible d'analyser cette distribution obtenue et ainsi d'effectuer un diagnostic.

\subsection{Discrimination de défauts}

Cette section vise à démontrer la pertinence de notre approche pour discriminer plusieurs types de défauts. Deux modes défaillants sont étudiés. Le premier est un court-circuit partiel d'un des pôles du rotor (18 spires sur 162), le deuxième est une excentricité dynamique.

Dans cette section, les mesures sont générées par un modèle 2D éléments finis. L'utilisation d'un modèle 2D s'explique par le fait qu'il est difficile de modéliser une excentricité dynamique en 3D même avec un modèle homogénéisé.

Les champs sont calculés sur un réseau de 24 capteurs. Il est à noter que des essais concluants ont également été obtenus avec des réseaux comportant moins de sondes. Après écriture du système [15] et résolution, nous obtenons trois répartitions de $\mathrm{T}$ correspondant aux trois cas.

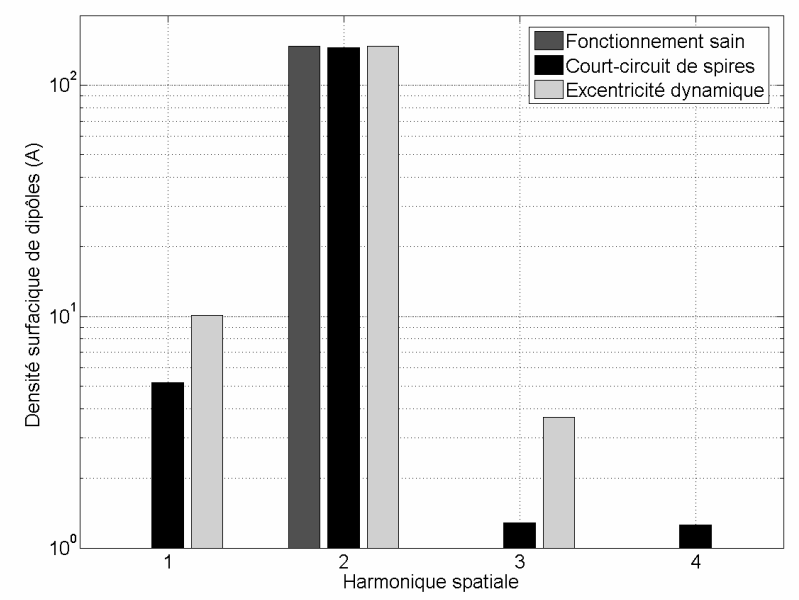

Figure 13. Décomposition spatiale de la distribution de dipôles $T$ dans l'entrefer pour les 3 cas (sain-court-circuit rotor-excentricité dynamique)

Pour le cas sain, seulement une raie spatiale (harmonique $p=2$ ) apparaît. Elle correspond aux 2 paires de pôles parfaitement symétriques. Pour l'excentricité dynamique, deux nouvelles raies apparaissent $(p=1$ et $p=3)$ de part et d'autre de la raie représentant la machine saine. Ces deux raies sont directement liées à l'équation [9] et peuvent être interprétées comme une modulation. Enfin, pour le court-circuit, une nouvelle raie, non présente dans les autres cas, apparaît $(p=4)$. 
Dans cet exemple, nous avons montré que notre méthode permet de discriminer plusieurs types de défaut sur un cas $2 \mathrm{D}$ simple. Nous allons voir qu'elle permet également de quantifier leur importance.

\subsection{Quantification du défaut}

Nous nous intéressons maintenant au cas où la machine présente un court-circuit au rotor (18 spires sur 162 sur un pôle soit à peu près $10 \%$ d'ampères-tours en moins). Les mesures sont générées par un modèle éléments finis 3D homogénéisé (section 3.3). Un réseau de 24 capteurs triaxiaux est utilisé. Nous obtenons après inversion une répartition dipolaire identifiée. Celle-ci peut être aisément comparée à celle obtenue directement lors de la modélisation éléments finis. La figure 14 présente, pour des raisons de lisibilité, la différence entre les dipôles de la machine saine et ceux de la machine fonctionnant avec un court-circuit de $10 \%$ sur un pôle.

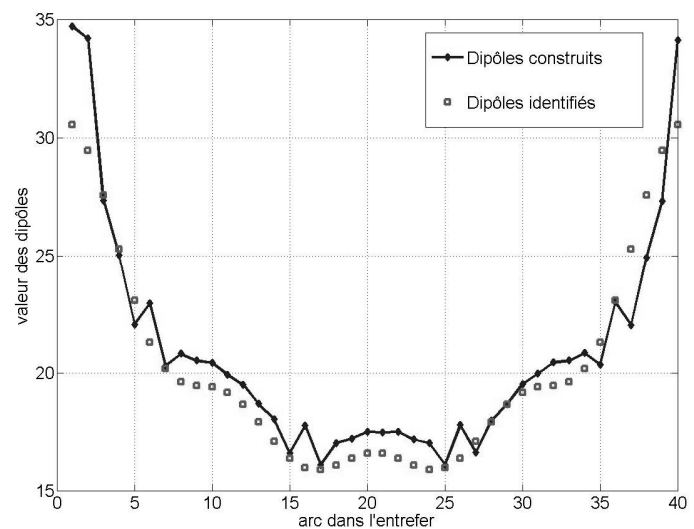

Figure 14. Comparaison entre la distribution calculée directement par la méthode des éléments finis et celle obtenue par identification (résolution de [15]) à partir des mesures du champ magnétique externe. Les courbes présentées sont la signature du défaut $\left(T_{\text {sain }}-T_{\text {défaut }}\right)$

Si nous analysons les courbes obtenues en figure 14, nous remarquons une bonne adéquation entre la courbe recherchée et la courbe identifiée par résolution du problème inverse.

La courbe identifiée est la grandeur $\mathrm{T}_{\text {sain }}-\mathrm{T}_{\text {défaut. }}$ Cette courbe est riche d'informations concernant l'importance du défaut et peut permettre de quantifier son importance.

Revenons à la définition de $\mathrm{T}$. Cette grandeur est la somme de $\mathrm{T}_{0}$ qui représente le nombre d'ampères-tours des bobinages et de $T_{\text {mat }}$ qui représente le flux circulant dans l'entrefer. Le flux dans l'entrefer étant conservatif, l'intégrale de $\mathrm{T}_{\text {mat }}$ sur la 
surface est par définition nulle. L'intégrale de $\mathrm{T}$ est donc égale à l'intégrale de $\mathrm{T}_{0}$ sur cette surface. Considérons maintenant les bobinages du rotor. Remarquons que si le rotor de la machine n'a pas de défaut, les Ampères-tours s'équilibrent parfaitement. L'intégrale de $\mathrm{T}_{0}$ est donc nulle. Par contre, si un court-circuit apparaît, celui-ci crée une dissymétrie de $\mathrm{T}_{0}$ et l'intégrale de cette grandeur sur la surface de l'entrefer est alors égale au nombre d'ampères-tours manquants multiplié par la surface de la boucle manquante.

Si nous reprenons maintenant l'équation [16] et que nous prenons son intégrale sur la surface de l'entrefer, nous constatons que les intégrales des termes sous le signe « somme » sont nulles. Il reste donc l'intégrale du terme $<\mathrm{T}>$. Nous obtenons donc :

$$
\mathrm{S}_{\text {défaut }} \times \mathrm{n}_{\text {court_circuit }} \mathrm{I}_{\text {rotor }}=\mathrm{S}_{\text {entrefer }} \times\langle\mathrm{T}\rangle
$$

où $\mathrm{n}_{\text {court-circuit }}$ est le nombre d'ampères-tours manquants.

Le paramètre $<\mathrm{T}>$ est identifié grâce à notre méthode. Nous pouvons donc quantifier l'importance du produit. En particulier, pour notre machine nous obtenons :

$$
\mathrm{S}_{\text {entrefer }} \times\langle\mathrm{T}\rangle=2.51 \mathrm{~A} \cdot \mathrm{m}^{2}
$$

D'autre part la signature a été obtenue avec un déficit de 324 ampères-tours dans une boucle qui présente un angle de $22^{\circ}$. Nous obtenons ainsi :

$$
\mathrm{S}_{\text {défaut }} \times \mathrm{n}_{\text {court_circuit }} \mathrm{I}_{\text {rotor }}=2.67 \text { A. } \mathrm{m}^{2}
$$

Les deux résultats obtenus présentent une très bonne concordance si on se rappelle du nombre d'approximations réalisées pour les obtenir. Ils démontrent d'une façon claire la pertinence de la méthode pour la quantification du défaut. Il est à noter que c'est le produit ampères-tours par la surface de la boucle correspondante qui est identifié. En particulier, la méthode ne peut actuellement pas discriminer une boucle d'une surface donnée parcourue par un courant I d'une autre boucle de surface deux fois moins importante parcourue par un courant 2I. Cette discrimination doit être possible en analysant la forme de la courbe 14. Cette analyse sera effectuée lors de travaux ultérieurs.

\section{Conclusions et perspectives}

\subsection{Conclusions}

Dans cet article, nous avons proposé une approche originale pour le diagnostic de défauts des machines électriques. Il s'agit de positionner des capteurs de champ 
magnétique à l'extérieur de la machine et d'analyser le flux fuite de celle-ci. Cette approche n'est pas nouvelle mais nous l'avons enrichie avec une méthodologie qui semble performante et prometteuse. Cette approche est basée sur la théorie des problèmes inverses. Dans un premier temps, nous avons développé un modèle éléments finis homogénéisé originalement appliqué à la problématique, nous permettant de simuler nos défauts, puis nous avons ensuite développé un nouveau modèle permettant de calculer très simplement le champ rayonné dans la machine. Ce modèle a ensuite été inversé et permet, à partir de mesures externes, de retrouver le champ circulant dans l'entrefer du dispositif avec une relativement bonne précision. Associé à un capteur de position du rotor, notre méthode permet de localiser et de quantifier l'importance du défaut. Nous avons démontré ce point avec un défaut en court-circuit mais la théorie peut être adaptée à d'autres types de défauts.

\subsection{Perspectives}

Evidemment, l'étude que nous présentons est numérique. En particulier, les champs magnétiques obtenus sur les capteurs ont été calculés par modèle. Ceci ne démontre donc pas entièrement la faisabilité de la méthode. Récemment, nous avons instrumenté la machine synchrone présentée dans cet article avec des capteurs de champ magnétique. Les mesures sont en cours et les résultats obtenus seront présentés dès que possible.

\section{Bibliographie}

Brunotte X., Meunier G., Imhoff J. F., "Finite elements solution of unbounded problems using transformations: a rigorous, powerful and easy solution", IEEE Transactions on Magnetics, vol. 28, March 1992, p. 1663-1666.

Chadebec O., Bui V.P., Granjon P., Rouve L-L., Le Bihan N., Coulomb J-L., "Rotor fault detection of electrical machines by low frequency magnetic stray field analysis", Proceedings of SDEMPED 2005 International Symposium on Diagnostic for Electric Machines, Power Electronics and Drives, Vienna, Austria, September 2005.

Chadebec O., Coulomb J-L., Janet F., “A review of Magnetostatic Moment Method”, IEEE Transactions on Magnetics, vol. 42, $\mathrm{n}^{\circ}$ 4, April 2006, p. 515-520.

Durand E., Magnétostatique, Paris, Masson et Cie, 1968.

Froidurot B., Rouve L-L., Foggia A., Bongiraud J-P., Meunier G., "Magnetic discretion of naval propulsion machines", IEEE Transactions on Magnetics, vol. 38, ${ }^{\circ} 2$, March 2002, p. $1185-1188$.

Henao H., Capolino G.A., Martis C., "On the stray flux analysis for the detection of the threephase induction machine faults", Proceedings of $38^{\text {th }}$ Industry Applications Conference, vol. 2, 2003, p. 1368-1373. 
Henneton A., Roger D., Napieralska E., "Study of electromagnetic phenomena contributing to external radiation of machines", International Conference on Electrical Machines, ICEM, Espoo, Finland, 2000.

Jerance N., Réseaux de reluctances et diagnostic de machines électriques, Thèse de doctorat de l'Institut national polytechnique de Grenoble, 2002.

Kildishev A.V., "Detection of magnetically unbalanced PM rotor in an assembled motor", Proceedings of Compumag Conference, 2003.

Mun Kwon O., Surussavadee C., Chari M.V.K., Salon S., "Analysis of the Far Field of Permanent-Magnet Motors and Effects of Geometric Asymmetries and Unbalance in Magnet Design”, IEEE Transactions on Magnetics, vol. 40, n² 2, March 2004.

Nandi S., Toliyat H.A., "Fault Diagnosis of Electrical Machines - A Review", Proceedings of IEEE-International Electric Machines and Drives Conference-IEMDC'99, Seattle, WA, USA, May 1999.

Schmerber L., Rouve L-L., Foggia A., "Original 2D cylindrical harmonics method for identification of the near magnetic stray field of an electrical motor", Proceedings of International Electric Machines and Drives Conference (IEMDC2005), 2005.

Timar P.L., Noise and Vibration of Electrical Machines, New York, Elsevier Science, 1989.

Wikswo Jr. J.P., Swinney K.R., "Scalar multipole expansions and their dipole equivalents”, Journal of Applied Physics, vol. 57, n 9, 1985, p. 4301-4308. 